\title{
MODELOS DE AMORTIZAÇÃO DE DÉFICITS ATUARIAIS EM FUNDOS DE PENSÃO
}

\section{UNFUNDED ACTUARIAL LIABILITY AMORTIZATION MODELS IN PENSION FUNDS}

\author{
JOSÉ ANGELO RODRIGUES \\ Professor Ms. dos Programas de Pós-Graduaçăo de Engenhiaria - COPPE \\ da Universidáde Federal do Rio de Janeiro - RJ \\ e đo MBA-Gestão Atuarial e Financeira da FIPECAFI - SP \\ E-mail: atuario@centroin.com.br
}

\section{RESUMO}

O financiamento de déficits atuariais em fundos de pensão sejam eles decorrentes da constituição insuficiente de ativos, revisão de premissas ou inclusão de participantes no plano com direitos não capitalizados, resume-se, gerálmente, ao uso de rendas financeiras temporárias, desprezando-se um conjunto de possibilidades oferecidas pela Matemática Financeira ou pela Matemática Atuarial. Este trabalho propõe sistematizar e demonstrar três conjuntos de métodos (financeiros e atuariais) disponíveis para atuários, administradores e patrocinadores, a fim de que possam efetuar a capitalização de tais insuficiências. Concluir-se-á demonstrando a diferença de velocidade de acumulação temporal de recursos para cobertura possibilitada pelos métodos analisados.

Palavras-chave: Fundos de Pensão; Déficits Atuariais; Métodos Atuariais de Custeio.

\section{ABSTRACT}

The financing of unfunded actuarial liabilities in pension funds, whether resulting from insufficient asset constitution, revision of actuarial premises or inclusion of participants in the plan with non capitalized interests, generally remains restricted to the use of $m$-year period certain annuity, ignoring a set of possibilities offered by financial or actuarial mathematics. This paper aims to demonstrate and systemize three groups of (financial and actuarial) methods, available to actuaries, managers and sponsors to capitalize such insufficiencies. Finally, we will demonstrate the different speed of temporal resource accumulation for the coverage made possible by the analyzed methods.

Keywords: Pension Fund; Unfunded Actuarial Liability; Actuarial Cost Methods. 


\section{INTRODUC̣ÃO}

Nos fundos de pensāo, não raro, ocorre a necessidade de que déficits decorrentes de serviços passados não fundados ou de insuficiências financeiras sejam cobertos por algum método dissociado ou não do Custo Normal adotado para financiamento do plano. Incluem-se, entre outros, os déficits: a) pelo reconhecimento de direitos sem a requerida contribuição na época própria; b) pela inclusão de participantes com direitos não capitalizados; c) pelo reconhecimento de insuficiências decorrentes da mudança de premissas e d) pela revisão de benefícios oferecidos pelo plano.

Este artigo busca mostrar alguns desses métodos. Não serão apuradas as insuficiências, mas, apenas, a partir de um valor dado como ideal para cobertura, evidenciar os métodos capazes de reduzir, gradualmente, a insuficiência e trazer equilíbrio atuarial ao plano. Serão abordados três métodos:

a) Modelo de Custo Suplementar Explícito, em que o método de financiamento dissocia-se do método atuarial de custeio corrente do plano;

b) Modelo de Custo Suplementar Implícito, no qual o método de financiamento utilizado e o método atuarial de custeio corrente do plano são idênticos e

c) Modelo de Amortização Direta, uma variação do Modelo de Custo Suplementar Explícito.

O quadro 10 mostra um resumo dos métodos tratados neste artigo.

O Método Explícito terá como ferramenta matemática apenas os conceitos da Matemática Financeira, ao passo que o Método Implícito aos conceitos da Matemática Financeira agregará processos atuariais. O Método Direto, embora descrito como uma variação do Modelo Suplementar Explícito, por tratar o financiamento como sendo a quitação do fluxo previdencial dos benefícios, obrigará o permanente reconhecimento atuarial, no plano, do valor sob amortização.

Todos os métodos tratados, neste artigo, têm vantagens e desvantagens relativamente a alguma particularidade do processo de saldamento do passivo a descoberto. Caberá aos administradores do passivo, particularizados os atuários, avaliar o conjunto das escolhas e proceder segundo as peculiaridades do plano sob financiamento.

\section{A DEFINIÇÃO DO PASSIVO A DESCOBERTO}

O cálculo do valor do passivo a descoberto, de um modo geral, decorre do cálculo individual de passivos, participante a participante. Sua definição agregada pode ser dada por $R M D_{t}=R M_{t}-A L_{t}$

em que: $R M D_{t}=$ reserva matemática a descoberto na época $t: R M_{t}=$ reserva matemática na época $t$ e $A L_{t}=$ ativo líquido na época $t$.

Sua definição individualizada será dada pela seguinte expressão:

$$
R M D_{t}=\sum_{k=1}^{n} R M D_{k, x} \quad=\sum_{k=1}^{n}\left(R M_{k, x}-A L_{k, x}\right)
$$

Para planos baseados em conceito coletivo de ativos, tem-se a diferenciação do modelo pelo valor do Ativo Líquido, que perde sua individualização.

$$
R M D_{t}=\left[\sum_{k=1}^{n} \sum_{x=\operatorname{Min}(x)}^{\operatorname{Max}(x)} R M_{k, x}\right]-A L_{t}
$$

a) Método Explicito $\left\{\begin{array}{l}\text { Amortizaçāo Constante } \\ \text { Termos Constantes } \\ \text { Termos Salariais }\end{array}\right.$
b) Método Implicito $\left\{\begin{array}{l}\text { Beneficio Acumulado }\left\{\begin{array}{l}\text { Valores Constantes } \\ \text { Percentuais Constantes }\end{array}\right. \\ \text { Beneficio Projetado }\left\{\begin{array}{l}\text { Valores Constantes } \\ \text { Percentuais Constantes }\end{array}\right.\end{array}\right.$

c) Método Direto

Quadro 1 Métodos Explícito, Implícito e Direto 
Para valores positivos de $R M D_{t}$, será atribuída a existência de um passivo a descoberto, estipulando-se um método temporal para cobertura. Como visto, a existência de valores positivos de $R M D$, pressupōe que o seu não saldamento ocasione, a cada ano, um excedente a descoberto, na forma:

$$
\Delta R M D_{t}=R M_{t+1}-E[R M D]_{t+1}
$$

em que: $\triangle R M D_{t}=$ variação do valor da reserva matemática a descoberto ocorrida no ano $t: R M D_{t+1}=$ reserva matemática a descoberto no ano $t+1$ e $E[R M D]_{t+1}=$ valor esperado da reserva matemática a descoberto no ano $t+1$.

Podem ser definidos os valores da fórmula (1) da seguinte forma:

$$
\begin{gathered}
E[R M D]_{t+1}=E[R M]_{t+1}=E[A L]_{t+1} \\
E[R M]_{t+1}=\left(R M_{t}+\sum_{k=1}^{n} C_{k, x}-\sum_{k=1}^{n} B_{k, x}\right) \cdot(1+i) \\
E[A L]_{t+1}=\left(A L_{t}+\sum_{k=1}^{n} C_{k, x}-\sum_{k=1}^{n} B_{k, x}\right) \cdot(1+i)
\end{gathered}
$$

em que: $C_{k, x}=$ contribuiçōes ao plano feitas pelos participantes (ativos e inativos): $B_{k x}=$ benefícios pagos pelo plano a participantes assistidos: $R M_{\mathrm{t}}=$ provisāo matemática no ano $t: A L_{\mathrm{t}}=$ ativo líquido no ano $t$ e $i=$ taxa de juros aplicável ao modelo.

Para sanar os efeitos do crescimento de $R M D_{t}$, precisa-se atribuir a $\triangle R M D_{t}$ valor igual a zero, na forma:

$$
\triangle R M D_{t}=0=R M D_{t}-\left(R M D_{t}-C A_{t}\right) \cdot(1+i)
$$

permitindo que $C A_{t}=\frac{R M D_{t} \cdot i}{(1+i)}$,

em que: $C A_{t}=$ complemento de amortização anual que estabiliza o déficit anual.

A determinação do valor de $C A_{\text {, }}$, entretanto, não é fator suficiente de cobertura do plano. Sua tarefa será, apenas, a de manter estável o déficit apurado. Ao valor de $C A$, será necessário acrescentar um valor que efetivamente amortize o déficit.

\section{A DEFINIÇÃO DOS CUSTOS ATUARIAIS}

Neste estudo será abordada, em diversas oportunidades, a constituição de Custos Normais e Custos Suplementares. Definindo-os:

- Custo Normal: refere-se ao valor pago ao plano pelo participante ou seu mantenedor, com base na idade de sua entrada no coletivo segurado, capaz de garantir os benefícios prometidos para a idade de elegibilidade. É função direta do método atuarial de financiamento adotado pelo plano.

- Custo Suplementar: refere-se ao valor pago ao plano pelo participante ou seu mantenedor, com base na idade de entrada no coletivo segurado (quando houver relação entre Custo Normal e Custo Suplementar), capaz de financiar a parte não constituída da provisão matemática e garantir os benefícios prometidos quando da elegibilidade. Pode ou não ter relação direta com o método de financiamento adotado pelo plano.

\section{MODELOS DE CUSTO SUPLEMENTAR EXPLÍCITOS'}

Como foi visto anteriormente, esses modelos estão baseados em princípios dissociados do modelo atuarial de financiamento adotado pelo plano. São apropriados a quaisquer tipos de métodos empregados no processo de financiamento de planos de benefícios justo por não precisarem estar associados a eles. Para os Modelos Explícitos, portanto, não serão definidos os Custos Normais para cada um dos métodos apresentados².

Não serão abordados, entretanto, três métodos pelos motivos que se seguem:

a) Método Price: assemelha-se ao Método de Termos Constantes, diferenciando-se, basicamente (e pouco). pelo valor do déficit remanescente gerado após cada amortização. Esse método tem o inconveniente de ter muitos de-

1 Săo denominados Modelos Explícitos. Externos ou Independentes em relação ao modelo corrente adotado pelo plano para financiamento do custo atuarial normal.

2 Nảo serão, nos modelos apresentados, considerados os éeitos da inflação. 
fensores e fortes opositores no que se refere ao senso de justiça financeira que sua aplicação pode resultar quando aplicado a contratos de amortização de dívidas ${ }^{3}$;

b) Método Americano: sob o ponto de vista do fundo de pensão funciona como um método de pagamento único. No período de amortizaçāo o plano de benefícios só estaria recebendo os juros relativos ao déficit apurado (mantendo o crescimento do déficit sob controle), ao passo que o valor do principal da dívida só seria quitado ao final do prazo estabelecido para amortizaçăo;

c) Método Sinking Fund: quanto ao objetivo (amortização no final do período) este método se assemelha ao Método Americano, diferenciando-se pelo critério de apropriação e cálculo dos juros relativos no período de amortização.

\subsection{Método de Amortização Constante}

Esse método prevê que a amortização do déficit se dará a dois termos. Como se viu anteriormente, $C A$, será a parte necessária para manter o déficit sob controle. Será necessário encontrar o segundo termo que, somado a $C A_{t}$, seja capaz de amortizar a insuficiência.

$$
C S_{t}=C A_{t}+A E_{t}
$$

em que: $C S_{t}=$ custo suplementar capaz de amortizar o passivo a descoberto na época $t$ e $A E_{t}=$ complemento de amortização efetiva na época $t$.

Sendo $C A_{t}$ a parte variável da amortização, $A E_{t}$ será a parte constante, capaz de amortizar em $n$ períodos o déficit definido. Assim, tem-se:

$$
\begin{gathered}
A E_{t+0}=A E_{t+1}=A E_{t+2}=A E_{t+3}=\ldots=A E_{t+n-1}=A E \\
R M D_{t}=n \cdot A E \\
A E=\frac{R M D_{t}}{n}
\end{gathered}
$$

em que: $R M D_{t}=$ Reserva Matemática a Descoberto na época t e $n=$ diferença temporal entre as idades inicial $\left(x_{a}\right)$ e final de amortização $(r)$, na forma $n=r-x_{a}$, considerado o número de termos da amortização.

Para definir o valor da amortização em um ano $k$ qualquer, ter-se-á:

$$
C A_{t+k}=\frac{R M D_{t+k} \cdot i}{(1+i)}
$$

Considerando as equações (3) e (4), define-se o valor de $R M D_{t}$ em $k$, na forma:

$$
\begin{gathered}
R M D_{t+k}=R M D_{t}-\underbrace{\left(A E_{t+0}+A E_{t+1}+A E_{t+2}+A E_{t+3}+\cdots+A E_{t+n-1}\right)}_{k \cdot A E} \\
=R M D_{t}-k \cdot A E
\end{gathered}
$$

ou ainda, considerando a equação (5):

$$
\begin{aligned}
R M D_{t+k} & =R M D_{t}-k \cdot \frac{R M D_{t}}{n} \\
= & R M D_{t} \cdot \frac{n-k}{n}
\end{aligned}
$$

em que: $k=$ número de parcelas de amortização feitas ou por fazer, sendo $k \leq n$.

A determinação do valor do Custo Suplementar, em $k$, será dada por meio das equações (5) e (6), na forma:

$$
\begin{aligned}
C S_{t+k}=C A_{t+k}+A E_{t+k} \\
=\frac{R M D_{t+k} \cdot i}{(1+i)}+\frac{R M D_{t+k}}{n}
\end{aligned}
$$

3 Ver Nogueira, José Jorge Moschiatti (2002) Tabela Price - Da Prova Documental e Precisa Elucidação do seu Anatocismo, Editora Servanda. 


$$
\begin{gathered}
=\frac{R M D_{t}}{n} \cdot\left[\frac{i}{(1+i)} \cdot(n-k)+1\right] \\
=\frac{R M D_{t}}{n} \cdot[d \cdot(n-k)+1]
\end{gathered}
$$
Assim:

O déficit remanescente deverá ser o valor resultante entre o déficit anterior e a parte constante calculada pelo modelo.

$$
R M D_{t+1}=R M D_{t}-\frac{R M D_{t}}{n}
$$

em que: $d=$ valor de desconto, sendo $d=i \cdot v=i /(1+i)$.

\subsection{Método de Termos Constantes}

Esse método é utilizado largamente na amortização de passivos atuariais, prevendo pagamentos constantes ao longo de um determinado prazo. Cada pagamento consiste na agregação das parcelas relativas à amortização e aos juros relativos ao período.

$$
R M D_{t}=C S_{i} \cdot \ddot{a}_{n_{i}}
$$

em que: $\left.\ddot{a}_{n}\right]_{i}=$ renda certa unitária, antecipada, de $n$ termos, à taxa $i$ de juros:

$$
\begin{gathered}
\ddot{a}_{n_{i}}=v^{0}+v^{1}+\cdot v^{2}+\cdots+v^{n-1} \\
=\sum_{t=0}^{n-1} v^{t}=\frac{1-v^{n}}{d}
\end{gathered}
$$

Uma vez determinado o período de financiamento do déficit, pode-se estabelecer:

$$
C S_{t}=\frac{R M D_{t}}{\ddot{a}_{n}}
$$

considerando que os pagamentos se darão de forma antecipada.

O valor do Custo Suplementar nos anos seguintes será dado por:

$$
C S_{t+0}=C S_{t+1}=C S_{t+2}=\cdots=C S_{t+n-1}
$$

Esse método permite monitorar o saldo da reserva não constituída numa data futura $t+k$, considerando, de forma retrospectiva, os valores já amortizados, na forma:

$$
R M D_{t+k}=R M D_{t} \cdot(1+i)^{k}-\left.C S_{t} \cdot \ddot{s}_{t+k}\right|_{i}
$$

considerando que o fator de acumulação

$$
\ddot{s}_{t+k}=\frac{(1+i)^{t+k}-1}{d}
$$

Pode-se escrever:

$$
R M D_{t+k}=R M D_{t} \cdot(1+i)^{k}=C S_{t} \cdot \frac{(1+i)^{t+k}-1}{d}
$$

em que: $\ddot{s}_{\bar{n} \backslash i}=$ montante de uma renda certa unitária, antecipada, de $n$ termos, à taxa $i$ de juros.

$O$ déficit remanescente deverá ser corrigido pela taxa de juros ajustada para o modelo. Dessa forma:

$$
R M D_{t+1}=\left(R M D_{t}-C S_{t}\right) \cdot(1+i)
$$

\subsection{Método de Termos Salariais}

Esse método guarda boa correlação com planos cujas contribuições estão associadas aos salários. Está associado ao crescimento salarial agregado (não decorrente de mérito) assumido pelo plano de benefícios. Seu objetivo é produzir uma 
estrutura de custeio semelhante ao custeio da folha salarial dos participantes. Tem, entretanto, o inconveniente de os salários poderem não ter a necessária correlação com o modelo salarial empregado para cálculo do financiamento da Reserva Matemática não constituída.

$$
\left.R M D_{t}=C S_{t} \cdot{ }^{(s)} \ddot{a}\right\rceil_{n i}
$$

em que:

${ }^{(s)} \ddot{a}_{n_{\mathrm{i}}}=$ renda certa, unitária, antecipada, temporária por n períodos, com crescimento salarial, considerando a taxa i de juros.

$$
\begin{aligned}
& \left.{ }^{(s)} \ddot{a}_{n}\right\rceil_{\mathrm{i}}=v^{0} \cdot(1+i s)^{0}+v^{1} \cdot(1+i s)^{1}+v^{2} \cdot(1+i s)^{2}+\cdots+v^{n-1} \cdot(1+i s)^{n-1} \\
& =\sum_{i=0}^{n-1} v^{t} \cdot(1+i s)^{t}=(1+i) \cdot \frac{1-v^{n} \cdot(1+i s)^{n}}{i+i s}
\end{aligned}
$$

is = inclinação da curva dos salários dos participantes ativos do plano, considerando inflação e produtividade incremento salarial.

Uma vez determinado o período de financiamento do déficit, pode-se estabelecer:

$$
C S_{t}=\frac{R M D_{t}}{{ }^{(s)} \ddot{a}_{n{ }_{i}}}
$$

O déficit remanescente será corrigido pela taxa de juros do modelo. Dessa forma:

$$
R M D_{t+1}=\left(R M D_{t}-C S_{t}\right) \cdot(1+i)
$$

Para o valor do Custo Suplementar no ano seguinte, ter-se-á:

$$
C S_{t+1}=C S_{t} \cdot(1+i s)
$$

\section{MODELOS DE CUSTO SUPLEMENTAR IMPLICITOS ${ }^{4}$}

Esses modelos estão baseados em princípios já utilizados pelo plano de benefícios para formação e manutenção de custeio dos benefícios. Seguem os princípios utilizados para formação do Custo Normal do plano. Cada um dos métodos utilizados para definição do Custo Normal de um plano deve ter seu método complementar implícito para formação da reserva suplementar. Para cada um dos métodos apresentados serão mostrados seus equivalentes ao Custo Normal do planos.

Cada um dos métodos, portanto, se diferenciará segundo as características econômicas e biométricas dos participantes. Todos os cálculos partem de elementos individualizados e. posteriormente, são agregados para determinação do seu valor global.

\subsection{Métodos de Custeio Atuariais}

Métodos de custeio atuariais representam modelos financeiro-atuariais da distribuição das prestações feitas ao plano por participantes e/ou patrocinadores ao longo do período laboral com o objetivo de constituir uma quantia suficiente para pagar os benefícios prometidos pelo plano. A cota de contribuição será distinta segundo o método de financiamento empregado. embora tais cotas sob diferentes métodos busquem constituir um mesmo valor para benefícios idênticos. Vide gráfico 10.

A diferença entre cotas para um mesmo tempo de contribuição sob diferentes métodos de financiamento se deve ao fato de que certos métodos privilegiam maiores custos em idades mais jovens e menores custos em idades maduras, ao passo que outros possam inverter tal escolha, ou ainda, distribuir tais cotas de forma equânime entre as idades.

Os modelos de distribuição de custos de um plano de benefícios podem ser concentrados em dois métodos distintos ${ }^{6}$ :

4 São denominados Modelos Implicitos. Internos ou Dependentes em relação ao modelo corrente adotado pelo plano para financiamento do custo atuarial normal 5 Não serão. nos modelos apresentados, considerados os efeitos da inflação.

6 Veja Tabela 3 sobre Métodos Atuariais de Financiamento. 


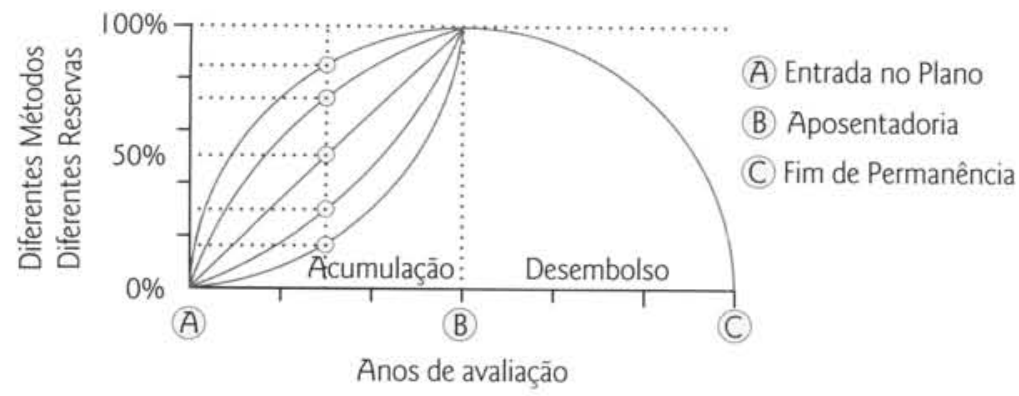

Gráfico 1

a) Método de Benefício Acumulado: aquele que predetermina a quantia do benefício a receber tornando cada fração do tempo de permanência no plano encarregada de gerar a acumulação da fração equivalente do benefício final;

b) Método de Benefício Projetado: aquele que predetermina a quantia do benefício a receber e o fraciona segundo um modelo financeiro-atuarial de renda pelo período de permanência no plano.

\subsection{Método de Benefício Acumulado (Modelo Geral)}

O Modelo Geral para o Custo Normal: o modelo geral para esse método procura definir a fraçāo anual do benefício futuro não constituído capaz de garantir o benefício integral prometido na idade de elegibilidade. Para um valor $b_{x^{\prime}}$ denominado fração do benefício, vai-se, segundo o método empregado, estabelecer seu valor de financiamento.

$$
C N_{x_{a}}=\underbrace{b_{x_{a}} \cdot \ddot{a}_{r}^{(m)}}_{\mathrm{A}} \cdot \underbrace{r_{r-x_{a}} p_{x_{a}}^{(T)} \cdot v^{r \rightarrow x_{a}}}_{\mathrm{B}}
$$

A - fração do benefício reconhecida na idade $x_{a}$ para ser recebida de forma vitalícia a partir da idade $r$ - retirement - de aposentadoria:

$B$ - fator de atualização atuarial relativo à idade de aposentadoria $e$ à idade $x_{a}$ de cálculo.

O Método de Benefício Acumulado divide-se em dois subgrupos com valores de $b_{x}$ diferenciados, a saber:

a) a Valores Constantes, em que a fração do benefício $b_{x}$ representa o benefício total $B_{r}$ na idade de aposentadoria distribuído para constituição entre as idades de aposentadoria $r$ e de entrada no plano $x_{e}$, na forma:

$$
b_{x_{a}}=\frac{B_{r}}{r-x_{e}}
$$

b) a Percentuais Constantes, em que a fração do benefício $b_{x}$ representa o benefício total $B_{f}$ na idade de aposentadoria dividido pela soma dos salários percebidos $S$ durante toda a permanência no plano, sob forma de percentual $k$, aplicáveis sobre os salários anuais $\mathrm{s}_{\mathrm{x}_{\mathrm{a}}}$, na forma:

$$
\begin{gathered}
b_{x_{a}}=k \cdot s_{x_{a}} \\
k=\frac{B_{r}}{S_{r}}
\end{gathered}
$$

O Modelo Geral para o Custo Suplementar: Relativamente ao Método de Benefício Acumulado, a Reserva Matemática a Descoberto, em uma determinada idade atingida, pode transformar-se em um benefício de aposentadoria $\left(B_{x_{a}}\right)$ não reconhecido naquela idade e não garantida pelo plano.

$$
\begin{aligned}
& R M D_{x_{a}}=R M_{x_{a}}-A L_{x_{a}} \\
= & B_{x_{a}} \cdot \ddot{a}_{r}^{(m)} \cdot{ }_{r-x_{a}} p_{x_{a}}^{(T)} \cdot v^{r-x_{a}}
\end{aligned}
$$

em que: $B_{x_{a}}=$ benefício acumulado para um participante de idade $x$. Representa a parte do benefício final não fundada para o participante; $\ddot{\alpha}_{r}^{(m)}=$ renda atuarial na idade $r$ de aposentadoria com decremento decorrente apenas de morte do participante; ${ }_{r-x_{a}} p_{x_{a}}^{(T)}=$ probabilidade de um participante de idade $x_{a}$ estar vivo ao completar a idade $r$ de aposentadoria e $x_{a}=$ data de início da amortização do custeio suplementar, ou data atual de avaliação atuarial. 
Pode-se simplificar os processos atuariais estabelecendo um valor (Z), capaz de representar o resultado obtido entre a idade de aposentadoria $(r)$, a idade de início da amortização acrescida dos anos determinados para amortização do déficit e a idade final de sobrevivência (caso o plano de financiamento se estenda por anos pós-laborais). Assim.

$$
Z=\operatorname{Min}\left\{\begin{array}{l}
r \\
x_{a}+n \\
\omega
\end{array}\right.
$$

em que: $r$ = se o período contributivo (e de amortização) se estender até a idade de aposentadoria; $x_{a}+n=$ se o período contributivo se estender da idade atingida até mais $n$ anos a contar dessa idade e $\omega=$ se o período contributivo se estender da idade atingida até a idade final de sobrevivência (ômega) prevista pela tábua de mortalidade.

Dessa forma, no decorrer do período de amortização, o valor de $R M D_{x_{a}}$ será:

$$
R M D_{x_{a}}=B_{x_{a}} \cdot \ddot{a}_{r}^{(m)} \cdot{ }_{r-x_{a}} p_{x_{a}}^{(T)} \cdot v^{r-x_{a}}
$$

ou ainda, considerando como $\mathrm{Z}$ a idade limite para amortização,

$$
R M D_{x_{a}}=\sum_{h=x_{a}}^{z} C S_{h} \cdot{ }_{h-x_{a}} p_{x_{a}}^{(T)} \cdot v^{h-x_{a}}
$$

Dessa forma, pode-se determinar que, com cada quota suplementar de amortização do déficit, se realiza um crédito suplementar do benefício não fundado na idade de início da amortizaçāo, na forma:

$$
\begin{gathered}
B_{x_{a}}=b_{x_{a}}^{s}+b_{x_{a}}^{s}+b_{x_{a}}^{s}+\cdots+b_{x_{a}}^{s} \\
B_{x_{a}}=\sum_{h=x_{a}}^{z} b_{h}^{s}
\end{gathered}
$$

Logo, o Custo Suplementar reconhece uma quantia anual adicional expressa por $b_{h}^{s}$ para garantir na idade de aposentadoria o benefício inicial prometido.

$$
C S_{x_{a}}=b_{x_{a}}^{s} \cdot \ddot{a}_{r}^{(m)} \cdot{ }_{z=x_{a}} p_{x_{a}}^{(T)} \cdot v^{z-x_{a}}
$$

em que: $b_{x_{a}}^{s}=$ fração nāo fundada do benefício final de aposentadoria.

Os Métodos apresentados neste artigo tomarão a idade $r$ para término do financiamento da Reserva Matemática a Descoberto.

\subsection{Método de Benefício Acumulado (Valores Constantes)}

A Definição do Custo Normal: O Custo Normal para esse método se constitui a partir da fração do benefício final projetado, na forma:

$$
C N_{x_{a}}=b_{x_{a}} \cdot \ddot{a}_{r}^{(m)} \cdot{ }_{r-x_{a}} p_{x_{a}}^{(T)} \cdot v^{r-x_{a}}
$$

sendo:

$$
\begin{gathered}
b_{x_{a}}=\frac{B_{x}}{r-x_{e}} \\
B_{r}=s_{r} \cdot g \\
s_{r}=s_{x_{a}} \cdot(1+i s)^{r-x_{a}}
\end{gathered}
$$

em que: $b_{x_{a}}=$ fração do benefício final de aposentadoria: $B_{r}=$ benefício na idade $r$ de aposentadoria: $s,=$ salário alcançado na idade $r$ de aposentadoria: $s_{x_{a}}=$ salário alcançado na idade $x_{a}$ de início do período de amortização: $x_{e}=$ idade de entrada no plano de benefícios e $g=$ regra de geração do benefício incidente sobre o salário na idade $r$ de aposentadoria.

Alguns outros valores precisam ser calculados como complemento ao Custo Normal:

a) Valor Atual do Benefício Futuro: 


$$
V A B F_{x_{a}}=B_{r} \cdot \ddot{a}_{r}^{(m)} \cdot{ }_{r-x_{a}} p_{x_{a}}^{(T)} \cdot v^{r-x_{a}}
$$

b) Reserva Matemática:

$$
R M_{x_{a}+n}=n \cdot\left(b_{x_{a}}+b_{x_{a}}^{s}\right) \cdot \ddot{a}_{r}^{(m)} \cdot{ }_{r-\left(x_{a}+n\right)} p_{x_{a}+n}^{(T)} \cdot v^{r-\left(x_{a}+n\right)}
$$

sendo:

$$
\begin{gathered}
b_{x_{a}}^{s}=\frac{B_{x_{a}}}{r-x_{a}} \\
B_{x_{a}}=\frac{R M D_{x_{a}}}{\ddot{a}_{r}^{(m)} \cdot{ }_{r-x_{a}} p_{x_{a}}^{(T)} \cdot v^{r-x_{a}}}
\end{gathered}
$$

em que: $V A B F_{x_{a}}=$ valor atual do benefício futuro de um participante da idade $x_{a}$ relativa ao início do período de amortizaçăo: $R M_{x_{a}}$ = reserva matemática de um participante na idade $x_{a}$ e $n=$ número de anos futuros para cálculo de função atuarial.

A Definição do Custo Suplementar: Compreende que o financiamento do valor de $R M D_{x_{a}}$ possa ser fracionado em $n$ partes entre as idades $r$ e $x_{a}$, na forma:

$$
C S_{x_{a}}=b_{x_{a}}^{s} \cdot \ddot{a}_{r}^{(m)} \cdot{ }_{r-x_{a}} p_{x_{a}}^{(T)} \cdot v^{r-x_{a}}
$$

Considerando:

$$
\begin{gathered}
b_{x_{a}}^{s}=\frac{B_{x_{a}}}{r-x_{a}} \\
B_{x_{a}}=\frac{R M D_{x_{a}}}{\ddot{a}_{r}^{(m)} \cdot{ }_{r-x_{a}} p_{x_{a}}^{(T)} \cdot v^{r-x_{a}}}
\end{gathered}
$$

por substituição, resultará:

$$
\begin{gathered}
C S_{x_{a}}=\frac{\frac{R M D_{x_{a}}}{\ddot{a}_{r}^{(m)} \cdot{ }_{r-x_{a}} p_{x_{a}}^{(T)} \cdot v^{r-x_{a}}}}{r-x_{a}} \cdot \ddot{a}_{r}^{(m)} \cdot{ }_{r-x_{a}} p_{x_{a}}^{(T)} \cdot v^{r-x_{a}} \\
=\frac{R M D_{x_{a}}}{r-x_{a}}
\end{gathered}
$$

O valor do déficit após a primeira amortização será dado pela expressăo:

$$
R M D_{x_{a}+1}=\left(R M D_{x_{a}}-C S_{x_{a}}\right) \cdot \frac{D_{x_{a}}}{D_{x_{a}+1}}
$$

O valor do Custo Suplementar no ano seguinte será dado pela expressão:

$$
C S_{x_{a}+1}=C S_{x_{a}} \cdot \frac{D_{x_{a}}}{D_{x_{a}+1}}
$$

O Custo Total será expresso pela soma dos custos normal e suplementar, na forma:

$$
C T_{x_{a}}=C N_{x_{a}}+C S_{x_{a}}
$$

\subsection{Método de Beneficio Acumulado}

(Percentuais Constantes)

A definição do Custo Normal: O Custo Normal para esse método se constitui a partir da fração do benefício final projetado como percentual do salário do participante, na forma:

$$
C N_{x_{a}}=b_{x_{a}} \cdot \ddot{a}_{r}^{(m)} \cdot{ }_{r-x_{a}} p_{x_{a}}^{(T)} \cdot v^{r-x_{a}}
$$


sendo:

$$
\begin{gathered}
b_{x_{a}}=k \cdot s_{x_{a}} \\
k=\frac{B_{r}}{S_{r}} \\
S_{r}=S_{x_{a}} \cdot(1+i s)^{x_{e}-x_{a}} \cdot \frac{(1+i s)^{r-x_{e}}-1}{i s}
\end{gathered}
$$

em que:

$k$ = percentual obtido a partir da relação entre o benefício projetado para a data de aposentadoria e os salários acumulados até a data de aposentadoria;

$S_{r}=$ Salários acumulados entre as datas de entrada no plano e aposentadoria, considerando o crescimento salarial;

$$
\begin{aligned}
& S_{r}=S_{x_{e}} \cdot(1+i s)^{1}+S_{x_{e}} \cdot(1+i s)^{2}+S_{x_{e}} \cdot(1+i s)^{3}+\cdots+S_{x_{e}} \cdot(1+i s)^{r-x_{e}} \\
& S_{r}=\sum_{t=1}^{r-x_{e}} S_{x_{e}} \cdot(1+i s)^{t}=S_{x_{e}} \cdot \frac{(1+i s)^{r-x_{e}}-1}{i s}
\end{aligned}
$$

Outros valores devem ser calculados para complemento ao cálculo do Custo Normal:

a) Valor Atual do Benefício Futuro:

$$
V A B F_{x_{a}}=B_{r} \cdot \ddot{a}_{r}^{(m)} \cdot{ }_{r-x_{a}} p_{x_{a}}^{(T)} \cdot v^{r-x_{a}}
$$

b) Reserva Matemática:

$$
R M_{x_{a}+n}=s_{x_{a}} \cdot \frac{(1+i s)^{n}-1}{i s} \cdot\left(k+k^{s}\right) \cdot \ddot{a}_{r}^{(m)} \cdot{ }_{r-\left(x_{a}+n\right)} p_{x_{a}+n}^{(T)} \cdot v^{r-\left(x_{a}+n\right)}
$$

sendo:

$$
\begin{gathered}
k^{s}=\frac{B_{x_{a}}}{S_{r}^{s}} \\
S_{r}^{s}=s_{x_{a}} \cdot \frac{(1+i s)^{r-x_{a}}-1}{i s}
\end{gathered}
$$

em que:

$k^{s}=$ percentual obtido a partir da relação entre o benefício acumulado até a idade de início de amortização e os salários acumulados entre as idades de início e final de amortização do déficit atuarial;

$S_{r}^{s}=$ salários acumulados entre as idades de início e final de amortização do déficit atuarial.

$$
\begin{aligned}
& S_{r}^{s}=s_{x_{a}}+s_{x_{a+1}}+s_{x_{a+2}}+\cdots+s_{x_{a+n-1}} \\
& S_{r}^{s}=\sum_{i=x_{a}}^{r-1} s_{h} \cdot(1+i s)^{\prime}=s_{x_{a}} \cdot \frac{(1+i s)^{r-x_{a}}-1}{i s}
\end{aligned}
$$

A Definição do Custo Suplementar: Compreende que o financiamento do valor de $R M D_{x_{a}}$ possa ser fracionado em $n$ partes entre as idades $r$ e $x_{a}$, considerando o crescimento dos salários no mesmo período, na forma:

$$
C S_{x_{a}}=b_{x_{a}}^{s} \cdot \ddot{a}_{r}^{(m)} \cdot{ }_{r-x_{a}} p_{x_{a}}^{(T)} \cdot v^{r-x_{a}}
$$


Considerando

$$
\begin{aligned}
b_{x_{a}}^{s} & =k^{s} \cdot S_{x_{a}} \\
k^{s} & =\frac{B_{x_{a}}}{S_{r}^{s}}
\end{aligned}
$$

por substituição:

$$
\begin{gathered}
C S_{x_{a}}=\frac{\frac{R M D_{x_{a}}}{\ddot{a}_{r}^{(m)} \cdot r_{r-x_{a}} p_{x_{a}}^{(T)} \cdot v^{r-x_{a}}}}{S_{r}^{s}} \cdot S_{x_{a}} \cdot \ddot{a}_{r}^{(m)} \cdot r_{r-x_{a}} p_{x_{a}}^{(T)} \cdot v^{r-x_{a}} \\
=\frac{R M D_{x_{a}}}{S_{r}^{s}} \cdot S_{x_{a}}
\end{gathered}
$$

O valor do déficit após a primeira amortização será dado pela seguinte expressāo:

$$
R M D_{x_{a+1}}=\left(R M D_{x_{a}}-C S_{x_{a}}\right) \cdot \frac{D_{x_{a}}}{D_{x_{a}+1}}
$$

O valor do Custo Suplementar no ano seguinte será dado pela equação a seguir:

$$
C S_{x_{a}+1}=C S_{x_{a}} \cdot \frac{D_{x_{a}}}{D_{x_{a}+1}} \cdot(1+i s)
$$

O Custo Total será expresso pela soma dos custos normal e suplementar, na forma:

$$
C T_{x_{a}}=C N_{x_{a}}+C S_{x_{a}}
$$

\subsection{Método de Benefício Projetado (Modelo Geral)}

Modelo Geral para o Custo Normal: O modelo geral de custeio procura definir o Custo Normal como fração resultante entre o custo futuro, na idade de entrada no plano, e uma renda temporária relativa ao período entre a idade de entrada no plano e a data de elegibilidade ao benefício.

$$
C N_{x_{a}}=b_{x} \cdot \ddot{a}_{r}^{(m)} \cdot{ }_{r-x_{e}} p_{x_{e}}^{(T)} \cdot v^{r-x_{e}}
$$

O Método de Benefício Projetado divide-se em dois subgrupos com valores de $b_{x}$ diferenciados, a saber:

a) a Valores Constantes, em que o benefício total $B_{r}$ na idade de aposentadoria é financiado por meio de uma renda atuarial temporária pelo período entre a idade de entrada no plano e a idade na data de elegibilidade, considerando os decrementos biométricos, com posição na data inicial $x_{e}$ de financiamento, na forma:

$$
\begin{aligned}
& b_{x}=\frac{B_{r}}{\ddot{a}_{x_{e}: r-x_{e}}^{(T)}} \\
& C N_{x_{a}}=\frac{B_{r}}{\ddot{a}_{x_{e}: r-x_{e}}^{(n)}} \cdot \ddot{a}_{r}^{(m)} \cdot{ }_{r-x_{e}} p_{x_{e}}^{(T)} \cdot v^{r-x_{e}} \\
& C N_{x_{a}}=\frac{V A B F_{x_{e}}}{\ddot{a}_{x_{e}: r-x_{e}}^{(T)}}
\end{aligned}
$$


b) a Percentuais Constantes, em que o benefício total $B_{r}$ na idade de aposentadoria é financiado por meio de uma renda atuarial temporária pelo período entre a idade de entrada no plano e a idade na data de elegibilidade, considerando o crescimento salarial e decrementos biométricos, com posição na data inicial $x_{e}$ de financiamento, na forma:

$$
\begin{aligned}
& b_{x}=\frac{B_{r}}{{ }^{(s)} \ddot{d}_{x_{e}}^{(T)} \frac{r_{r-x_{e}}}{t}} \\
& b_{x_{e}}=\frac{B_{r}}{{ }^{(s)} \ddot{a}_{x_{e}}^{(T)} \overline{: r-x_{e}}} \\
& C N_{x_{e}}=\frac{B_{r}}{{ }^{(s)} \ddot{a}_{x_{e}: \overline{r-x_{e}}}^{(T)}} \cdot \ddot{a}_{r}^{(m)} \cdot_{r-x_{e}} p_{x_{e}}^{(T)} \cdot v^{r-x_{e}} \\
& C N_{x_{e}}=\frac{V A B F_{x_{e}}}{{ }^{(s)} \ddot{d}_{x_{e}}^{(T)} \overline{r r-x_{e}}} \\
& C N_{x_{a}}=\frac{V A B F_{x_{e}}}{{ }^{(s)} \ddot{a}_{x_{e}: r-x_{e}}^{(T)}} \cdot(1+i s)^{x_{a}-x_{e}}
\end{aligned}
$$

Modelo Geral para o Custo Suplementar: Esse método permite o financiamento do passivo a descoberto pelo tempo de permanência do participante no plano ou por períodos determinados. O passivo a descoberto relativo a uma idade atingida se financia atuarialmente pelo período que vai da idade atingida até a idade de aposentadoria (compreenda-se que este limite poderá ser determinado arbitrariamente), por meio de aportes suplementares, cujo valor atuarial deve ser igual ao valor desse passivo.

$$
R M D_{x_{a}}=\sum_{h=x_{a}}^{r-1} C S_{h} \cdot{ }_{r-h} p_{h}^{(T)} \cdot v^{r-h}
$$

O valor de $R M D_{x_{a}}$ deverá ser financiado, atuarialmente, durante o período que se estende da idade atingida até a idade de aposentadoria ou até findarem os anos determinados para amortizaçăo do déficit. Dessa forma, no decorrer do período de amortização, o valor de $R M D_{x_{a}}$ será dado pela equação:

$$
R M D_{x_{a}}=B_{x_{a}} \cdot \ddot{a}_{r}^{(m)} \cdot{ }_{r-x_{a}} p_{x_{a}}^{(T)} \cdot v^{r-x_{a}}
$$

ou ainda, considerando como $\mathrm{Z}$ a idade limite para amortização,

$$
R M D_{x_{a}}=\sum_{h=x_{a}}^{z} C S_{h} \cdot{ }_{h-x_{a}} p_{x_{a}}^{(T)} \cdot v^{h-x_{a}}
$$

O valor de Z será determinado como o menor resultado obtido entre a idade de aposentadoria, a idade atingida acrescida dos anos determinados para a amortização e a idade final de sobrevivência (caso o plano preveja contribuições póslaborais). Assim:

$$
Z=\operatorname{Min}\left\{\begin{array}{l}
r \\
x_{a}+n \\
\omega
\end{array}\right.
$$

Dessa forma, com cada quota suplementar de amortização se realiza um crédito suplementar do benefício complementar não garantida na idade atingida, na forma:

$$
\begin{gathered}
B_{x_{a}}=b_{x_{a}+0}^{s}+b_{x_{a}+1}^{s}+b_{x_{a}+2}^{s}+b_{x_{a}+3}^{s}+\cdots+b_{r-1}^{s} \\
B_{x_{a}}=\sum_{h=x_{a}}^{Z} b_{h}^{s}
\end{gathered}
$$


Logo, o Custo Suplementar reconhece uma quantia anual adicional expressa por $b_{h}^{s}$ para garantir na idade de aposentadoria o benefício inicial prometido.

$$
C S_{x_{a}}=b_{x_{a}}^{s} \cdot \ddot{a}_{r}^{(m)} \cdot{ }_{z-x_{a}} p_{x_{a}}^{(T)} \cdot v^{z-x_{a}}
$$

Os Métodos apresentados, neste artigo, tomarão a idade $r$ para término do financiamento da Reserva Matemática a Descoberto.

\subsection{Método de Benefício Projetado (Valores Constantes)}

A Definição do Custo Normal: O Custo Normal será obtido pela fração resultante entre o Valor Atual do Benefício Futuro inicial e uma renda atuarial temporária. considerando os múltiplos decrementos, pelo período entre a idade de entrada no plano e a data de aposentadoria, na forma:

$$
\begin{gathered}
C N_{x_{e}}=b_{x} \cdot \ddot{a}_{r}^{(m)} \cdot{ }_{r-x_{e}} p_{x_{e}}^{(T)} \cdot v^{r-x_{e}} \\
C N_{x_{e}}=\frac{B_{r}}{\ddot{a}_{x_{e}}^{(T)} \overline{r-x_{e}}} \cdot \ddot{a}_{r}^{(m)} \cdot{ }_{r-x_{e}} p_{x_{e}}^{(T)} \cdot v^{r-x_{e}} \\
V A B F_{x_{e}}=B_{r} \cdot \ddot{a}_{r}^{(m)} \cdot{ }_{r-x_{e}} p_{x_{e}}^{(T) \cdot} \cdot v^{r-x_{e}} \\
C N_{x_{e}}=\frac{V A B F_{x_{e}}}{\ddot{a}_{x_{e}: \overline{r-x_{e}}}^{(T)}} \\
C N_{x_{a}}=\frac{V A B F_{x_{e}}}{\ddot{a}_{x_{e}}^{(T)} \overline{r-x_{e}}}
\end{gathered}
$$

em que: $V A B F_{x_{e}}=$ valor atual dos benefícios futuros na idade $x_{e}$ inicial de entrada no plano de benefícios $e^{\ddot{a}_{x_{e}}} \overline{\left.r-x_{e}\right]}=$ renda atuarial a partir da idade de entrada $x_{e}$, temporária por $r-x_{e}$ períodos (todo o período de permanência no plano de benefícios), considerando todos os decrementos utilizados.

Outros valores devem ser calculados para complemento ao cálculo do Custo Normal:

a) Valor Atual do Benefício Futuro:

$$
V A B F_{x_{a}+n}=\frac{V A B F_{x_{a}}}{{ }_{n} p_{x_{a}}^{(T)} \cdot v^{n}}
$$

b) Reserva Matemática:

$$
R M_{x_{a}+n}=V A B F_{x_{a}+n} \cdot \frac{\ddot{a}_{x_{a}: n}^{(T)}}{\ddot{a}_{x_{a}: r-x_{a}}^{(T)}}
$$

A Definição do Custo Suplementar: Esse método em muito se assemelha ao Método Explícito a Termos Constantes, diferenciando-se pelo tratamento atuarial aplicado.

$$
C N_{x_{a}}=\frac{R M D_{x_{a}}}{\ddot{a}_{x_{a}}^{(T)} \overline{r-x_{a}}}
$$

O valor do déficit após a primeira amortizaçāo será dado pela seguinte equação:

$$
R M D_{x_{a}+1}=\left(R M D_{x_{a}}-C S_{x_{a}}\right) \cdot \frac{D_{x_{a}}}{D_{x_{a}+1}}
$$


O valor do Custo Suplementar nos anos seguintes será dado pela expressão:

$$
C S_{x_{a}+0}=C S_{x_{a}+1}=C S_{x_{a}+2}=C S_{x_{a}+3}=\cdots=C S_{r-1}
$$

O Custo Total será expresso pela soma dos custos normal e suplementar, na forma:

$$
C T_{x_{a}}=C N_{x_{a}}+C S_{x_{a}}
$$

\subsection{Método de Benefício Projetado (Percentuais Constantes)}

A Definição do Custo Normal: O Custo Normal será obtido pela fraçāo resultante entre o Valor Atual do Benefício Futuro inicial e uma renda atuarial temporária, considerando os múltiplos decrementos e crescimento salarial, pelo período entre a idade de entrada no plano e a data de aposentadoria, na forma:

$$
\begin{aligned}
& C N_{x_{e}}=b_{x} \cdot \ddot{a}_{r}^{(m)} \cdot{ }_{r-x_{e}} p_{x_{e}}^{(T)} \cdot v^{r-x_{e}} \\
& b_{x}=\frac{B_{r}}{(s) \ddot{d}_{x_{e}}^{(T)} \overline{r-x_{e}}} \\
& V A B F_{x_{e}}=B_{r} \cdot \ddot{a}_{r}^{(m)} \cdot{ }_{r-x_{e}} p_{x_{e}}^{(n)} \cdot v^{r-x_{e}} \\
& C N_{x_{e}}=\frac{V A B F_{x_{e}}}{{ }^{(s)} \ddot{a}_{x_{e}}^{(T)}: r-x_{e}} \\
& C N_{x_{a}}=\frac{V A B F_{x_{a}}}{(s) \ddot{d}_{x_{e}}^{(T)} \overline{r-x_{e}}} \cdot(1+i s)^{x_{a}-x_{e}}
\end{aligned}
$$

Outros valores devem ser calculados para complemento ao cálculo do Custo Normal:

a) Valor Atual do Benefício Futuro:

$$
V A B F_{x_{a}+n}=\frac{V A B F_{x_{a}}}{{ }_{n} p_{x_{a}}^{(T)} \cdot v^{n}}
$$

b) Reserva Matemática:

$$
R M_{x_{a}+n}=V A B F_{x_{a}+n} \cdot \frac{{ }^{(s)} \ddot{a}_{x_{a}}^{(T)}: n}{\left({ }^{(s)} \ddot{a}_{x_{a}}^{(T)}: r-x_{a}\right.}
$$

A Definição do Custo Suplementar: Esse método busca encontrar um percentual incidente sobre o salário projetado capaz de financiar o valor de $R M D_{x_{a^{*}}}$ O estabelecimento desse percentual $\left(k^{s}\right)$ deve ser oriundo da expressão a seguir:

$$
C S_{x_{a}}=k^{s} \cdot s_{x_{a}}
$$

Sendo:

$$
k^{s}=\frac{R M D_{x_{a}}}{s_{x_{a}} \cdot{ }^{(s)} \ddot{a}_{x_{a}}^{(T)} \overline{r-x_{a}}}
$$

por substituição:

$$
C S_{x_{a}}=\frac{R M D_{x_{a}}}{s_{x_{a}} \cdot{ }^{(s)} \ddot{a}_{x_{a} \div r-x_{a}}^{(T)}} \cdot s_{x_{a}}
$$




$$
C S_{x_{a}}=\frac{R M D_{x_{a}}}{{ }^{(s)} \ddot{a}_{x_{a}}^{(T)} \overline{r-x_{a}}}
$$

O valor do déficit após a primeira amortização será dado pela seguinte expressão:

$$
R M D_{x_{a}+1}=\left(R M D_{x_{a}}-C S_{x_{a}}\right) \cdot \frac{D_{x_{a}}}{D_{x_{a}+1}}
$$

O valor do Custo Suplementar no ano seguinte será dado pela seguinte equação:

$$
C S_{x_{a}+1}=C S_{x_{a}} \cdot(1+i s)
$$

O Custo Total será expresso pela soma dos custos normal e suplementar, na forma:

$$
C T_{x_{a}}=C N_{x_{a}}+C S_{x_{a}}
$$

\section{MODELO DE AMORTIZAÇÃO DIRETA}

O Modelo de Amortização Direta representa, provavelmente, a maneira mais suave de financiamento de passivos atuariais, sendo bastante apropriado para patrocinadores que precisem transferir encargos atuariais para seus fundos de pensāo.

Considere que um determinado grupo de empregados (ativos ou aposentados) esteja sob contrato, vinculado a ter, no período pós-laboral, vencimentos pagos pelo ex-empregador. A capacidade de pagar tais benefícios pode nāo representar dificuldade para o fluxo financeiro do empregador, mas a antecipação desses encargos pode se mostrar bastante onerosa.

Esse método de amortização possibilita o reconhecimento do passivo e seu financiamento em longo prazo, segundo a longevidade dos participantes (pode ser estabelecido um prazo menor que a expectativa de vida do participante mais jovem, exigindo, dessa forma, um incremento para formação de um ativo excedente para antecipação de pagamentos futuros). Devem ser estabelecidos os seguintes passos:

a) Reconhecimento do Passivo Atuarial pelo fundo de pensão:

- Participantes Ativos:

$$
{ }^{(a)} R M D_{x_{a}}=s_{x_{a}} \cdot(1+i)^{r-x_{a}} \cdot g \cdot \ddot{a}_{r}^{(m)} \cdot{ }_{r-x_{a}} p_{x_{a}}^{(T)} \cdot v^{r-x^{a}} \quad\left\{x_{e} \leq x_{a}<r\right\}
$$

- Para todos os participantes ativos:

$$
\left.{ }^{(a)} R M D_{x_{a}}=\sum_{k=1}^{n} s_{x_{a} \cdot k} \cdot(1+i)^{r-\left(x_{\alpha} k\right)} \cdot g \cdot \ddot{a}_{r}^{(m)} \cdot{ }_{r-\left(x_{\alpha} k\right.}\right)_{x_{\alpha^{k}} k}^{(T)} \cdot v^{r-\left(x_{\alpha} k\right)}
$$

- Participantes Assistidos:

$$
{ }^{(r)} R M D_{x_{a}}=B_{x_{a}} \cdot \ddot{a}_{r}^{(m)}\left\{r \leq x_{a}<\omega\right\}
$$

- Para todos os participantes assistidos:

$$
{ }^{(n)} R M D_{x_{a}}=\sum_{k=1}^{n} B_{x_{a}, k} \cdot \ddot{a}_{x_{a}, k}^{(m)}
$$

- Para todos os grupos (ativos e assistidos):

$$
R M D_{x_{a}}={ }^{(a)} R M D_{x_{a}}+{ }^{(r)} R M D_{x_{a}}
$$

b) Reconhecimento do Custo Suplementar, pelo patrocinador, a ser pago ao plano:

- Participantes ativos:

$$
{ }^{(a)} C S_{x_{a}}=S_{x_{a}} \cdot{ }^{(c)} t c
$$


- Para todos os participantes ativos:

$$
{ }^{(a)} C S_{x_{a}}=\sum_{k=1}^{n} s_{x_{a}, k} \cdot{ }^{(\%)} t c
$$

- Participantes assistidos:

$$
{ }^{(n)} C S_{x_{a}}=B_{x_{a}}
$$

- Para todos os participantes assistidos:

$$
{ }^{(r)} C S_{x_{a}}=\sum_{k=1}^{n} B_{x_{a}, k}
$$

c) Reconhecimento do fluxo de contribuições anuais devido ao plano:

$$
\begin{gathered}
C S_{x_{a}}=\sum_{k=1}^{n} B_{x_{a}, k}+\sum_{k=1}^{n} s_{x_{a}, k} \cdot\left({ }^{(\%)} t c\right. \\
C S_{x_{a}}=\sum_{k=1}^{n} B_{x_{a}, k}+s_{x_{a}, k} \cdot{ }^{\left.()_{0}\right)} t c
\end{gathered}
$$

em que: ${ }^{(\%)} t c=$ taxa de contribuição, sob forma percentual, definida para o plano de benefícios: $n=$ número de participantes do plano e $k=$ um dado participante do plano.

Esse método consiste no reconhecimento do passivo atuarial pelo fundo de pensão e, pelo patrocinador, no reconhecimento do seu compromisso de pagar benefícios previdenciais relativos ao passivo reconhecido até a extinçāo do último participante. O pagamento dos benefícios, sob responsabilidade do plano, embora subvencionado pelo patrocinador, no tempo, deverá amortizar a Reserva Matemática de Benefícios Concedidos. Todos os cálculos acima deverão ser repetidos anualmente, refazendo-se o cálculo de reservas e custos de fluxos de caixa previdenciais.

Nesse Método é importante saber que, em valor presente, o fluxo de caixa dos benefícios pagos pelo patrocinador será igual ao valor da Reserva Matemática, sendo a vantagem de sua adoçāo a de que o déficit deverá ser saldado apenas quando da extinçăo do grupo por falecimento. Assim, traça-se as curvas das Reservas Matemáticas e dos pagamentos equivalentes, na forma expressa pela equação a seguir:

$$
V A B F_{x_{a}}=\sum_{h=x_{a}}^{\infty} B_{h} \cdot{ }_{r-h} p_{h}^{(r)} \cdot v^{r-h}
$$

As curvas de Reserva Matemática e Fluxo de Caixa Atuarial terão o desempenho mostrado pelo gráfico $2 \mathbf{0}$.

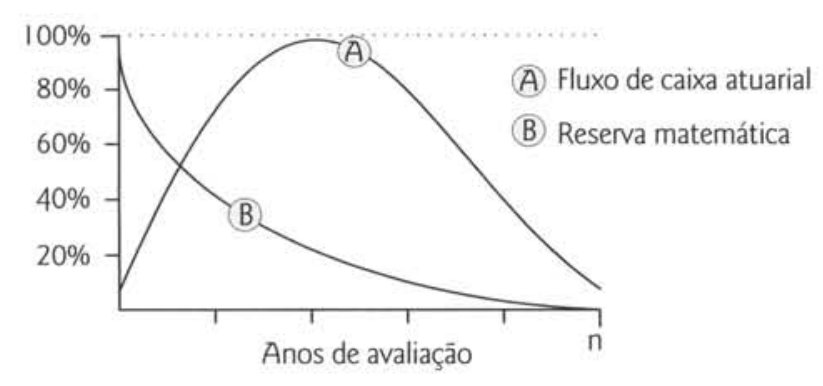

Gráfico 2 


\section{CONCLUSÕES}

Algumas considerações sobre os Métodos de Financiamento de Déficits Atuariais:

a) Os diferentes métodos apresentados (Explícitos. Gráfico $3 \mathbf{0}$ e Implícitos. Gráfico $4 \mathbf{0}$ ) produzem valores bastante diferenciados durante o período de amortização, devendo o fundo de pensão e a patrocinadora ou o associado. avaliar o método a ser utilizado segundo a sua capacidade de pagar prestaçōes.

Os Métodos Explícitos (Gráfico $5 \mathbf{0}$ ), no que se refere à velocidade de amortização são mais eficientes. São convexos em relação aos Métodos Implícitos (Gráfico 6 O). como se pode observar nos gráficos comparativos a seguir.

b) O Método Explícito de Termos Salariais e o Método Implícito de Benefício Projetado a Percentuais Constantes têm valores de amortização diferentes, embora produzam percentuais de acumulação idênticos, ou seja, fazem chegar ao plano de benefícios valores financeiros diferentes, embora amortizem percentuais idênticos da dívida. Vide Tabela 10.

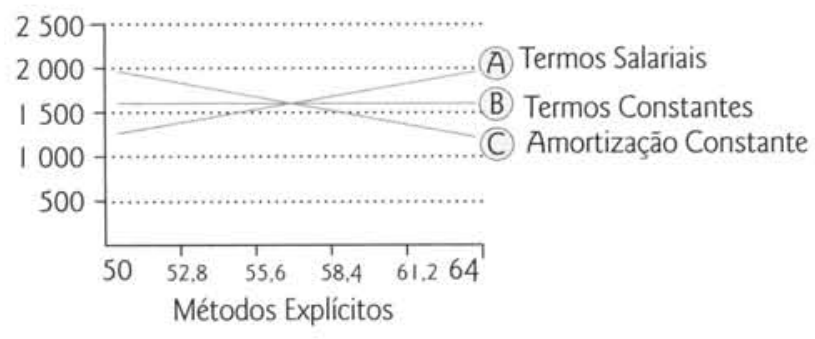

Gráfico 3

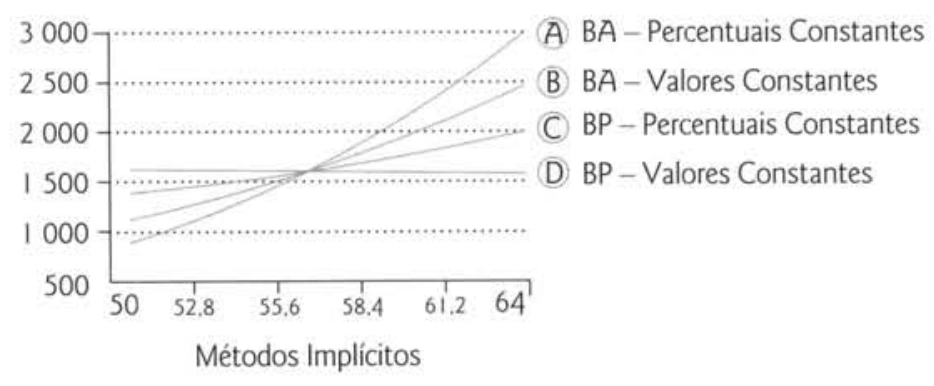

Gráfico 4

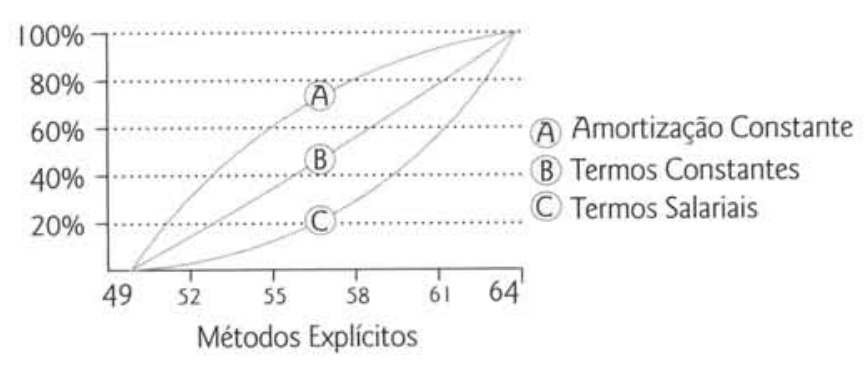

Gráfico 5

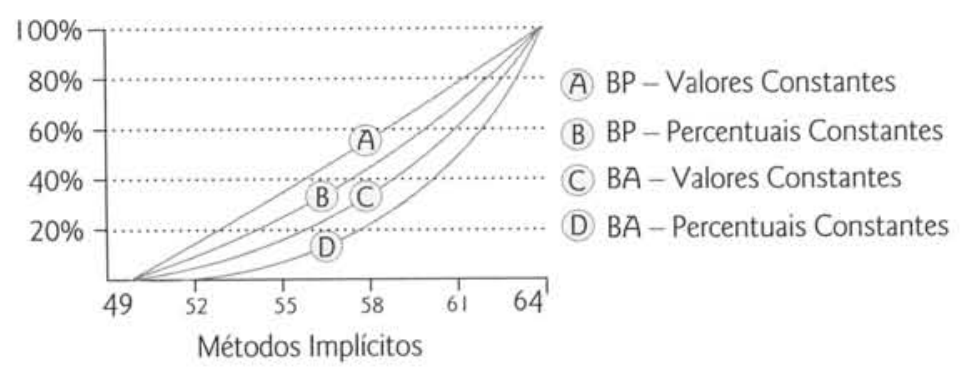


Tabela 1 Utilizado um modelo para financiamento em 15 anos (participante de 50 anos), para um financiamento de R\$17.000,00

\begin{tabular}{|c|c|c|c|c|c|c|c|}
\hline & \multicolumn{3}{|c|}{ Métodos Financeiros } & \multicolumn{4}{|c|}{ Métodos Financeiro-Atuariais } \\
\hline & \multirow{2}{*}{$\begin{array}{c}\text { Amortização } \\
\text { Constante }\end{array}$} & \multirow{2}{*}{$\begin{array}{c}\text { Termos } \\
\text { Constantes }\end{array}$} & \multirow{2}{*}{$\begin{array}{l}\text { Termos } \\
\text { Salariais }\end{array}$} & \multicolumn{2}{|c|}{ Benefício Acumulado } & \multicolumn{2}{|c|}{ Beneficio Projetado } \\
\hline & & & & $\begin{array}{c}\text { Valores } \\
\text { constantes }\end{array}$ & $\begin{array}{l}\text { Percentuais } \\
\text { Constantes }\end{array}$ & $\begin{array}{c}\text { Valores } \\
\text { Constantes }\end{array}$ & $\begin{array}{l}\text { Percentuais } \\
\text { Constantes }\end{array}$ \\
\hline 0 & $0,00 \%$ & $0,00 \%$ & $0,00 \%$ & $0,00 \%$ & $0,00 \%$ & $0,00 \%$ & $0,00 \%$ \\
\hline 1 & $8,28 \%$ & $6,67 \%$ & $5,58 \%$ & $4,40 \%$ & $3,59 \%$ & $6,67 \%$ & $5,58 \%$ \\
\hline 2 & $16,32 \%$ & $13,33 \%$ & $11,29 \%$ & $9,05 \%$ & $7,47 \%$ & $13,33 \%$ & $11,29 \%$ \\
\hline 3 & $24,14 \%$ & $20,00 \%$ & $17,15 \%$ & $13,95 \%$ & $11,67 \%$ & $20,00 \%$ & $17,15 \%$ \\
\hline 4 & $31,72 \%$ & $26,67 \%$ & $23,16 \%$ & $19,12 \%$ & $16,21 \%$ & $26,67 \%$ & $23,16 \%$ \\
\hline 5 & $39,08 \%$ & $33,33 \%$ & $29,31 \%$ & $24,58 \%$ & $21,12 \%$ & $33,33 \%$ & $29,31 \%$ \\
\hline 6 & $46,21 \%$ & $40,00 \%$ & $35,62 \%$ & $30,34 \%$ & $26,44 \%$ & $40,00 \%$ & $35,62 \%$ \\
\hline 7 & $53,10 \%$ & $46,67 \%$ & $42,09 \%$ & $36,43 \%$ & $32,20 \%$ & $46,67 \%$ & $42,09 \%$ \\
\hline 8 & $59,77 \%$ & $53,33 \%$ & $48,72 \%$ & $42,87 \%$ & $38,44 \%$ & $53,33 \%$ & $48,72 \%$ \\
\hline 9 & $66,21 \%$ & $60,00 \%$ & $55,51 \%$ & $49,68 \%$ & $45,21 \%$ & $60,00 \%$ & $55,51 \%$ \\
\hline 10 & $72,41 \%$ & $66,67 \%$ & $62,48 \%$ & $56,89 \%$ & $52,55 \%$ & $66,67 \%$ & $62,48 \%$ \\
\hline 11 & $78,39 \%$ & $73,33 \%$ & $69,62 \%$ & $64,52 \%$ & $60,52 \%$ & $73,33 \%$ & $69,62 \%$ \\
\hline 12 & $84,14 \%$ & $80,00 \%$ & $76,93 \%$ & $72,62 \%$ & $69,18 \%$ & $80,00 \%$ & $76,93 \%$ \\
\hline 13 & $89,66 \%$ & $86,67 \%$ & $84,43 \%$ & $81,20 \%$ & $78,59 \%$ & $86,67 \%$ & $84,43 \%$ \\
\hline 14 & $94,94 \%$ & $93,33 \%$ & $92,12 \%$ & $90,31 \%$ & $88,84 \%$ & $93,33 \%$ & $92,12 \%$ \\
\hline 15 & $100,00 \%$ & $100,00 \%$ & $100,00 \%$ & $100,00 \%$ & $100,00 \%$ & $100,00 \%$ & $100,00 \%$ \\
\hline
\end{tabular}

c) Para os Métodos Implícitos, mostra-se que a definição dos custos Normal e Suplementar passa por definir o valor de $b_{x}$ como elemento definidor na constituição do Benefício Final a ser financiado pelo participante ou mantenedor. Pode-se resumir. a partir desse fato, seu quadro resumo de operaçōes, conforme apresentado na Tabela $2 \boldsymbol{0}$.

d) Como se viu, foram resumidos os Métodos Atuariais como sendo de Benefício Acumulado e Benefício Projetado. Na Tabela $3^{7} \boldsymbol{O}$. discriminaram-se os métodos atuariais clássicos e seu enquadramento em relação ao benefício ${ }^{8}$.

Tabela 2 Custos Normal e Suplementar - para o Método de Benefício Acumulado sāo apresentadas as fraçōes de cálculo e para o Método de Benefício Projetado apresenta-se o custo Normal e Suplementar em fórmula final

\begin{tabular}{|c|c|c|}
\hline Custo Normal & Custo Suplementar & Método Atuarial \\
\hline$\frac{B_{r}}{r-x_{e}}$ & $\frac{B_{x_{d}}}{r-x_{a}}$ & $\begin{array}{l}\text { Beneficio Acumulado } \\
\text { Valores Constantes }\end{array}$ \\
\hline$k \cdot s_{x_{a}}$ & $k^{5} \cdot s_{x_{a}}$ & $\begin{array}{l}\text { Beneficio Acumulado } \\
\text { Percentuais Constantes }\end{array}$ \\
\hline$\frac{V A B F_{x_{e}}}{a_{x_{e}}^{(n)} \overline{r-x_{e}}}$ & $\frac{R M D_{x_{a}}}{a_{x_{a}}^{(n)} \overline{r-x_{a}}}$ & $\begin{array}{l}\text { Benefício Projetado } \\
\text { Valores Constantes }\end{array}$ \\
\hline$\frac{V A B F_{x_{e}}}{\left(s \ddot{a}_{x_{e}}^{(T)} \frac{T}{r-x_{e}}\right.} \cdot(1+i s)^{x_{a}-x_{e}}$ & $\frac{R M D_{x_{a}}}{(s) \ddot{a}_{x_{a}}^{(r)} \overline{\left.r-x_{a}\right)}}$ & $\begin{array}{c}\text { Beneficio Projetado } \\
\text { Percentuais Constantes }\end{array}$ \\
\hline
\end{tabular}

7 Resumo da Table 10-1 in Winklevoss. H.E. (1993) Pension Mathematics with Numerical Illustrations. p. 143

8 Esta classificação está em acordo com a terminologia empregada pelo JCPT - Joint Committee on Pension Terminology (1981) e adotada pelas seguintes entidades: AAA - American Academy of Actuaries, Conference of Actuaries in Public Practice. SoA. Society of Actuaries e ASPA - American Society of Pension Actuaries. 
Tabela 3 Métodos Atuariais - destaca-se que apenas ao Credito Unitário (não projetado) se pode creditar o Método de Benefício Acumulado

\begin{tabular}{|c|c|}
\hline Método Atuarial & Termo Genético \\
\hline Crédito Unitário (não projetado) & $\begin{array}{l}\text { Método de Benefício Acumulado com Custo Suplementar } \\
\text { Explícito; }\end{array}$ \\
\hline Crédito Unitário (projetado) & $\begin{array}{l}\text { Método de Beneficio Projetado com Custo Suplementar } \\
\text { Explicito (somente para a versão a Valores Constantes); }\end{array}$ \\
\hline Método da Idade à Entrada Normal & $\begin{array}{l}\text { Método de Beneficio Projetado com Custo Suplementar } \\
\text { Explicito (somente para a versâo a Valores Constantes); }\end{array}$ \\
\hline Método da Idade Atingida Normal & $\begin{array}{l}\text { Método de Benefício Projetado (Valores Constantes ou } \\
\text { Percentuais Constantes); }\end{array}$ \\
\hline Método de Prêmio Nivelado Individual & $\begin{array}{l}\text { Método de Benefício Projetado (Valores Constantes ou } \\
\text { Percentuais Constantes); }\end{array}$ \\
\hline Método do Passivo Inicial Congelado & $\begin{array}{l}\text { Método de Beneficio Projetado (Valores Constantes e Per- } \\
\text { centuais Constantes); }\end{array}$ \\
\hline Método de Idade Atingida Normal (Grupal) & $\begin{array}{l}\text { Método de Benefício Projetado (Valores Constantes e Per- } \\
\text { centuais Constantes). Cálculos grupais; }\end{array}$ \\
\hline Método Agregado & $\begin{array}{l}\text { Método de Benefício Projetado com Custo Suplementar } \\
\text { Implicito (Valores Constantes ou Percentuais Constantes). } \\
\text { Cálculos grupais; }\end{array}$ \\
\hline Método Agregado Individual & $\begin{array}{l}\text { Método de Benefício Projetado com Custo Suplementar } \\
\text { Implicito (Valores Constantes ou Percentuais Constantes). } \\
\text { Cálculos individualizados. }\end{array}$ \\
\hline
\end{tabular}

\section{Bibliografias}

BUTCHER, M.V.; NESBITT, C. J.. Mathematics of Compound Interest. USA: Ultich Books, 1971.

ESTEBAN, J. I. de La Peña. Planes de Previsión Social. Espanha: Ediciones Pirámide, 2000.

Amortización Del Déficit en La Exteriorización de Compromisos para un Plan de Prestación Definida. IEF-A, España, s/d.

MATIAS, W.F.; GOMES, J. M.. Matemática Financeira. Brasil: Atlas, 1996.

RODRIGUES, J. A.. Introduçāo à Gestāo de Risco Atuarial. Brasil: Mimeo, 2005.

VILANOVA, W.. Algebra Financeira. Biblioteca de Ciências Sociais, Brasil, 1980.

WINKLEVOSS, H. E.. Pension Mathematics with Numerical Illustrations. USA, 1993.

\section{NOTA - Endereço do autor}

Universidade Federal do Rio de janeiro COPPE - Prédio do Centro de Tecnologia Bloco G, Sala 101, Cidade Universitária Rio de Janeiro - RJ

21.945-970 